\title{
Editorial
}

\section{Clinical Factor 2014}

\author{
Richard Balon \\ Departments of Psychiatry and Behavioral Neurosciences and of Anesthesiology, Wayne State University School of \\ Medicine, Detroit, Mich., USA
}

As in the previous 4 years [1-4], I am presenting my subjective selection, this time of 16 (primary) articles published in the previous year (2014) that I believe may provide some information to help the reader improve his/ her clinical practice. As I noted before, the idea was originally proposed by Giovanni Fava [5] with the goal of selecting papers that I would subjectively perceive as having a high 'clinical factor' - the degree and extent to which a journal article provides information to the clinician that may improve his/her practice. As I wrote before about the selected articles: 'Many may find them useful, some will find them less useful or even useless. I am certainly biased, as is anybody else. I also cannot review all psychiatric journals - I focused only on most of the major ones. If there is no article selected from a certain major psychiatric journal, it does not necessarily mean that I have not reviewed it or skimmed through that journal. It may mean that I did not find, in my opinion, anything clinically very interesting' [1]. I view myself first and foremost as a clinician (though academically oriented) and I am, as most clinicians are, looking for a way to help patients and for guidance in the treatment of my patients, and thus looking for new, innovative or newly validated treatments. I am sure I missed a number of valuable articles, but as noted, this is a subjective selection.

\section{Psychosis and Schizophrenia}

The relationship between schizophrenia and cannabis use is a complex one. Cannabis use has been considered deleterious to patients with schizophrenia. How- ever, not much is known about the impact of cannabis use on the prognosis of schizophrenia (yet a negative impact has been suspected). Manrique-Garcia et al. [6] in their cohort study used a unique set of data of 50,087 Swedish male conscripts who were followed from 1973 to 2007 (permission to use this data set was granted by the Stockholm Regional Ethical Review Board). These men were conscripted during 1 year (1969-1970) for compulsory military training. All of them completed 2 self-report nonanonymous questionnaires at the time of conscription. The first questionnaire focused on social upbringing, friendships, relationships, attitudes and adjustment at school and work. The second questionnaire concerned the use of alcohol, tobacco, drugs and substance use. Cannabis use was categorized as a dichotomous variable: ever used versus never used. Those presenting with psychiatric symptoms during psychological assessment were referred to a psychiatrist, and if a psychiatric disorder was found, it was diagnosed according to the ICD-8. The authors identified $401(0.8 \%$ of the cohort) subjects as suffering from schizophrenia during the follow-up period. Of those, 44 did not respond to the question on drug abuse at baseline. Among the remaining 357 men, 72 (20\%) reported having a history of cannabis use. Interestingly, schizophrenia patients without a history of cannabis use were more likely to have paranoid schizophrenia than the ever users (17 vs. $8 \%$ ). Importantly, schizophrenia patients with a history of cannabis use had (a) a higher median duration of the first hospital episode (59 vs. 30 days), (b) a higher median rate of readmission (10 vs. 4 times), a higher total number of hospital days (547 vs. 184), in-

\section{KARGER 125}

(c) 2015 S. Karger AG, Base

0033-3190/15/0846-0330\$39.50/0
Richard Balon, MD

Department of Psychiatry and Behavioral Neurosciences Tolan Park Building, Third Floor

3901 Chrysler Service Dr., Detroit, MI 48201 (USA)

E-Mail rbalon@wayne.edu 
creased odds of having more than 20 readmissions (3.1, 95\% CI 1.3-7.3) and increased odds of hospital admissions lasting more than 2 years $(2.4,95 \%$ CI 1.1-7.4). All that after controlling for the diagnosis of personality disorder, family socioeconomic position, IQ scores, civil status, place of residence, risky use of alcohol and use of other drugs. This clearly demonstrated a higher burden of disease among those schizophrenia patients with a history of cannabis use. The study had several limitations (only men, no information about the continuation of cannabis use, no information about treatment), yet it suggests that not only can cannabis increase the risk of schizophrenia, but that it could impact the course and prognosis of the illness. (This study was supported by grants from the Swedish Council for Working Life and Social Research Project and from the Stockholm County Council.)

In an interesting study, Harrow et al. [7] attempted to address an important issue in the treatment of schizophrenia - the lack of evidence of long-term ( $>3$ years) treatment with antipsychotic medication and whether antipsychotics reduce or eliminate psychosis in longterm treatment. At a relatively early phase of their disorder, 139 patients were assessed prospectively and then reassessed at 5 or 6 subsequent follow-ups over the next 20 years by trained interviewers who were not informed of their diagnosis or of the results of their previous followup examinations. The assessments included structured research (SADS) and functioning interviews, and occurred at index hospitalization and at 2, 4.5, 7.5, 10, 15 and 20 years after the index hospitalization. There were 70 schizophrenia spectrum disorder patients (61 schizophrenia and 9 schizoaffective disorder) and 69 nonschizophrenia patients (38 psychotic bipolar disorder and 31 psychotic unipolar depression). There was no single uniform treatment plan that would apply to all patients. At the 2-year assessment, $62 \%$ of schizophrenia spectrum disorder patients were prescribed antipsychotic medication with or without other medications and another $9 \%$ were prescribed other medications. More than $70 \%$ of schizophrenia patients on continuously prescribed antipsychotics experienced psychotic activity at 4 or more of 6 follow-ups over 20 years. Patients not prescribed antipsychotics showed significantly less psychotic activity (0.05). This data suggests that after the first few years antipsychotic medications do not eliminate or reduce the frequency of psychosis in schizophrenia, or reduce the severity of postacute psychosis. As this was an observational and naturalistic study and the sample was small, the results are certainly not unambiguous. Nevertheless, these results raise many questions about the long-term treatment, e.g. how many patients really reach remission and whether antipsychotics lose their efficacy during long-term treatment. As evidence suggests that longterm outcome for schizophrenia has not improved from the preantipsychotic era [8], it seems that we need to reevaluate our approach to the long-term management of this disorder. (This work was supported, in part, by US Public Health Service Grants, a grant from the NIMH and a grant from the Foundation for Excellence in Mental Health.)

Most schizophrenia patients show some cognitive deficit compared to the general population and probably to their premorbid level of functioning. Cognitive remediation has been provided to schizophrenia patients for decades; however, neither the cognitive remediation programs nor their results have been uniform and uniformly beneficial. In another study of cognitive remediation, Ostergaard Christensen et al. [9] examined the effects of a 16-week cognitive remediation program (NEUROCOM) combined with early intervention versus early intervention alone in a randomized trial of 117 patients with a first episode of psychosis. Investigators, but not patients or cognitive trainers, were blinded during the baseline, posttraining and follow-up assessment - patients were instructed not to tell what type of intervention they received. A total of 98 patients $(83.8 \%)$ participated in posttraining assessment (4 months) and 92 (78.6\%) in the 12-month follow-up assessment. Most patients were on medication. No effects were found on the primary outcome measure - functional capacity - as measured by the University of California San Diego Performance Skills Assessment scale. At the follow-up, the intervention group retained significant improvement in the verbal learning domain $(\mathrm{p}<0.05)$, and also significant improvements were observed in the working memory domain $(\mathrm{p}=$ $0.01)$ and PANSS positive symptoms $(\mathrm{p}=0.04)$. While there were some immediate and long-term effects on cognitive functioning, symptoms and even self-esteem, there was no effect on functional capacity. Interestingly, the cognitive remediation intervention seemed to enhance the effects of early interventions in psychotic symptomatology. (This study was supported by the Danish Medical Research Council, the Lundbeck Foundation, an unrestricted grant by Bristol Myers Squibb, Sygekassernes Helsefond, the University of Copenhagen Faculty of Health Sciences, Board of Region H, Mental Health Centre Copenhagen and the Research Fund of Aarhus University Hospital, Risskov.) 


\section{Mindfulness Intervention}

Studies showed the effect of mindfulness meditation training on well-being and outcome of various mental and physical diseases, including substance use disorders. The high degree of relapse to substance use disorders is a serious problem, as $40-60 \%$ of individuals with substance use disorders relapse within 1 year. Relapse prevention (RP) therapy has been widely studied and implemented. While helpful, RP has various shortcomings, such as focus on avoidance-based goals versus approach-based goals and on controlling causes of negative affect or craving versus learning to tolerate these states. It also places relatively little emphasis on individual needs. Thus, Bowen et al. [10] studied the effects of mindfulness-based relapse prevention (MBRP) and RP and compared them to treatment as usual (TAU). MBRP is a group-based psychosocial aftercare which integrates evidence-based practices from mindfulness-based intervention and cognitive-behavioral RP approaches. TAU provided 12-step programming and psychoeducation. Participants were randomly assigned to 8 weeks of group session of MBRP (100 attended $\geq 1$ session, 79 included in 12-month analysis), RP (88 attended $\geq 1$ session, 64 in 12-month analysis) and TAU (92 attended $\geq 1$ session, 64 in 12-month analysis). Primary outcome measures included relapse to drug use and heavy drinking and frequency of substance use in the past 90 days. Measures used included self-report of relapse as well as urinalysis and alcohol drug screenings. Assessments were done at baseline and at 3,6 and 12 months. When compared to TAU, both MBRP and RP significantly lowered the risk of relapse to substance use and heavy drinking, and among those who used substances, significantly fewer days of substance use and heavy drinking at the 6-month follow-up. RP showed an advantage over MBRP in time to first drug use; however, MBRP subjects reported significantly fewer days of substance use and significantly decreased heavy drinking when compared to RP and TAU at the 12-month follow-up. The limitations included using the self-report data and limited urinalysis data, in addition to difference in therapist training and assigned homework. However, the long-term MBRP effect in reducing drug use and heavy drinking is an important finding. It may be explained, as the authors point out, by the subjects' improved ability to recognize and tolerate discomfort associated with craving or negative affect. (This study was supported by the National Institutes of Health/ National Institute on Drug Abuse, some support from the National Institute of Alcohol Abuse and Alcoholism, and Recovery Center of King County.)

\section{Cognitive-Behavioral Therapy}

Cognitive-behavioral therapy (CBT) has been refined, combined with other treatment modalities and compared to various treatments to gather more data on its usefulness, feasibility and efficacy. Wiersma et al. [11] examined the efficacy of the Cognitive-Behavioral Analysis System of Psychotherapy (CBASP), a stage-specific psychotherapeutic intervention for chronic depression. In a multisite (3 outpatient clinics in the Netherlands) 52week controlled trial, they randomized 67 patients to CBASP and 72 patients to care as usual (CAU). CBASP consisted of a mean of 24 sessions administered biweekly for the first month, then weekly for 3 months and then gradually every 2 weeks and finally once a month. CAU consisted of a mean of 23 sessions, also as CBT sessions lasting 45 min each, mostly CBT (53\% of patients) and interpersonal therapy (25\%), and the remainder of the patients was treated with various supportive therapies. Pharmacotherapy was provided in both arms and was supported by clinical management. Some patients refused medication and received therapy only. The primary outcome measure was the Inventory for Depressive Symptomatology (IDS) Self-Report. CBASP and CAU did not statistically differ on the IDS after 8,16 and 32 weeks. However, in week 52, the mean IDS scores were significantly different, the CBASP scores dropped from 40.8 to 24.1 , and the CAU scores dropped from 43.8 to $32.0(\mathrm{p}=0.05)$. In addition, the CBASP patients who completed the study were more likely to respond (41.2 vs. $18.9 \%, \mathrm{p}=0.01)$ and to remit $(26.0$ vs. $9.4 \%)$ than CAU patients. The limitations of this study included a significant proportion of noncompleters $(25 \%)$ and a difference in therapist training (CBASP received vigorous training). (This study was supported by a grant from the Netherlands Organization for Health Research and Development.)

Cognitive therapy (CT) is frequently combined with antidepressant treatment, either in a parallel or a sequential fashion. Both modalities are efficacious in the treatment of depression, but not all patients achieve remission and even fewer patients achieve recovery on antidepressants alone. Hollon et al. [12] sought to determine whether combining CT with antidepressants enhances recovery when treatment is personalized. They randomized 452 patients with chronic or recurrent major depressive disorder (MDD) to either antidepressant medication alone (225 patients) or to antidepressant medication combined with CT. The trial was conducted at 3 outpatient centers in the USA. Treatment was continued for up to 42 months, 
until recovery was achieved. The main outcome measures were a modified version of the 17-item Hamilton Rating Scale for Depression and the Longitudinal Interval Follow-Up Evaluation. Antidepressant treatment was administered according to a principle-based algorithm that could involve up to 4 different classes of antidepressants and any commonly used augmenting or adjunctive agent. Dosages were raised rapidly and kept at maximum tolerated levels for at least 4 weeks. The CT protocol called for 50 -min sessions twice weekly for at least the first 2 weeks, at least weekly thereafter during acute treatment, and then at least monthly during continuation. Combined treatment enhanced the rate of recovery when compared to antidepressant treatment alone $(72.5$ vs. $62.5 \%, \mathrm{p}=$ 0.01 ; or 165 vs. 140 recovered patients). The advantage of combined treatment was, however, limited to patients with severe, nonchronic MDD. Remission rates did not differ significantly either as a main effect of treatment or as an interaction with severity or chronicity. Patients in the combined group dropped out less frequently and reported fewer side effects (probably because they experienced less time in an episode of MDD). Patients with comorbid personality disorder took longer to achieve recovery. Thus, interestingly, the CT + antidepressant medication combination effect in enhancing recovery was mostly limited to patients with severe, nonchronic depression, which should probably be the target population for this combination. (This study was supported by grants from the National Institute of Mental Health, and Wyeth Pharmaceuticals provided venlafaxine and Pfizer Inc. sertraline for the trial. The funding sources had no role in the design, conduct of the study, data collection, management, analysis, interpretation of the data and preparation and submission of the manuscript.)

In their previous report, Leichsenring et al. [13] found that both CBT and psychodynamic psychotherapy were superior to waiting list control with regard to remission and response of patients with social anxiety disorder (SAD). This study was actually part of the Social Phobia Psychotherapy Network Study A1, a large multicenter randomized controlled trial conducted in Germany, comparing CBT and psychodynamic psychotherapy in SAD. There was a statistically significant difference in favor of CBT with regard to remission but not response. As this was a relatively short study (about 6 months) and there are few studies examining the long-term outcome of psychotherapy in this disorder, the authors decided to follow the participant status over the ensuing 24 months. In their recent report, Leichsenring et al. [14] report results of assessing these patients (207 treated with psycho- dynamic psychotherapy and 209 with CBT) 6, 12 and 24 months after the end of therapy. Primary outcome measures were rates of remission and response. Interestingly, there were no statistically significant differences between these two therapies. The response rates for both groups were quite similar: for CBT after treatment and at 6,12 and 24 months, the rates were $63,72,70$ and $69 \%$, for psychodynamic psychotherapy (same assessment points) 58 , 65,64 and $69 \%$. Remission rates for the CBT group were $38,44,44$ and $39 \%$, for psychodynamic psychotherapy 28 , 37,37 and $38 \%$. Interestingly, the early results [15] suggesting superiority of CBT over psychodynamic psychotherapy in SAD disappeared in the long-term follow-up, and both therapies fared basically the same. This raises questions about the nonspecificity of the psychotherapy effect in SAD or the possibility of some patients benefiting from one treatment more than from the other. (This study was supported by a grant from the German Federal Ministry of Education and Research.)

\section{Technology Applied to (Psycho)Therapy (Is It Still Psychotherapy?)}

Various Internet and other technology-based therapies have been introduced lately, presumably mostly because of the barriers to treatment. Two groups studied Internet-based CBT (ICBT) for obsessive-compulsive disorder (OCD). Herbst et al. [15] conducted a small study of 34 OCD patients who were randomized into an immediate start group or to a waiting list control group (WLCG). The treatment lasted 8 weeks, the WLCG actually also received treatment, but delayed by 8 weeks. The intervention consisted of Interned-based writing therapy with therapeutic interaction. All communication between the participants and therapists took place exclusively via the Internet. The treatment consisted of 14 sessions (twice a week with the exception of the first and the last week), which were based on established and evidencebased CBT manuals and methods. Treatment was conducted by 3 experienced CBT therapists (at least 4 years of therapeutic experience). The outcome measures used were the Yale-Brown Obsessive Compulsive Scale (YBOCS), Obsessive-Compulsive Inventory-Revisited, Patient Global Impression of Improvement Scale, Beck Depression Inventory-II and Internet Specific Questions. Patients were assessed at baseline, before the intervention (i.e. after the waiting period for the WLCG), directly after the treatment, 8 weeks and 6 months after completing the treatment. OCD symptoms were significantly improved 
in the treatment group compared to the WLCG after the first period (effect sizes of Cohen's $d=0.82$ for Y-BOCS SR and 0.87 for Obsessive-Compulsive Inventory-Revisited). The effect of treatment remained stable at the 6-month follow-up. The limitations were considerable: small sample, no active control group and heterogeneous sample. Nevertheless, this study suggests that ICBT may be useful in the treatment of OCD and that its effects may be comparable to those of traditional face-to-face CBT. (This study was supported by a grant from the German Research Foundation.)

In their follow-up study, Andersson et al. [16] investigated the long-term efficacy of ICBT with therapist support for OCD without an Internet-based booster program. This was a continuation of their previous randomized clinical trial of ICBT for OCD [17] in which 101 OCD patients received either 10 weeks of ICBT $(n=50)$ or supportive therapy $(\mathrm{n}=51)$. The ICBT consisted of written self-help material and homework assignments that were reviewed by an online therapist. The main components of this acute ICBT were psychoeducation, cognitive restructuring, exposure and response prevention and an RP program. At the posttreatment assessment in this original study, $60 \%$ of the ICBT group showed significant improvement on the Y-BOCS, while only $6 \%$ of participants in the control group improved significantly. In the follow-up study [16], the participant sample consisted of the original cohort that received ICBT and of the original control group that received supportive therapy, but was then crossed to ICBT. So, in the follow-up, the entire sample was originally treated with ICBT. Participants who completed a 4-month follow-up were randomly assigned to the booster group $(\mathrm{n}=47)$ or a present control group ( $\mathrm{n}=46$, no booster treatment). The 'booster' consisted of a self-help text with worksheets and an integrated e-mail system on a secured online platform. This treatment format was identical with the treatment in the previous study with consecutive modules followed by homework exercises and frequent written contact with an online therapist. Concurrent use of medications was allowed. Assessments (using the Y-BOCS) were done at 7, 12 and 24 months. The booster group had a significant mean reduction in OCD symptoms compared to the control condition from the booster baseline (4 months) to 7 months, but not at 12 and 24 months. The booster group improved significantly in terms of general functioning at 7,12 and 24 months and had fewer relapses. Adding the booster treatment clearly improved long-term outcome and prevented relapse in some OCD patients receiving ICBT. (This study was supported by Stockholm County Coun- cil, Karolinska Institutet, the Swedish Research Council and the Swedish Society of Medicine.)

Another 2 studies examined the delivery of CBT over the Internet in depression. In a small study of 62 depressed patients, Wagner et al. [18] compared therapistsupported Internet-based intervention $(\mathrm{n}=32)$ and faceto-face intervention $(\mathrm{n}=30)$. Online and face-to-face intervention groups received a brief, 8-week CBT program for depression, involving 7 modules: introduction, behavioral analysis, planning of activities, daily structure, life review, cognitive restructuring, social competence and relapse prevention. The online intervention was given as a guided intervention with intensive therapist contact. The therapist time involved responding to texts, requiring 20-50 min per text, depending on the therapist experience with Internet-based therapies. Each writing assignment lasted $45 \mathrm{~min}$, and patients were given 2 assignments each week of the 8-week study. Therapists responded with written feedback within 1 working day. Patients in the face-to-face group attended 1-hour weekly treatment sessions with their allocated psychologist and were also given weekly homework assignments (e.g. daily structured diaries, negative thoughts log). The outcome measures (primary: Beck Depression Inventory-II scores; secondary: suicidal ideation, anxiety, hopelessness, automatic thoughts, treatment satisfaction) were collected before treatment, after treatment and at the 3-month follow-up. Interestingly, there were no significant betweengroup differences for any of the pre- to posttreatment measurements. At the posttreatment assessment, significant symptom changes occurred in both intervention groups compared to before the intervention symptomatology. At the 3-month follow-up, the online group remained stable, while the face-to-face group showed significantly worsened depressive symptomatology. These findings were limited by the small size of both groups. Nevertheless, this study provides another evidence that online CBT intervention for depression is working and is probably as effective as comparable face-to-face intervention. It is important to realize that ICBT interventions require therapist involvement and time, too. (This study was supported by the Selo Foundation, Switzerland.)

Phillips et al. [19] tested the effectiveness of a computerized CBT intervention (MoodGYM course developed by the Australian National University). Their study was a 2-arm, parallel randomized controlled trial with a 5-week intervention period and follow-up at 6 and 12 weeks. The trial was designed to be administered mainly online. The MoodGYM course allowed participants to proceed at their own pace over five 1-hour modules, usually taken 
weekly. The control group was given a selection of 5 selfhelp in mental illness websites to use. The study was designed to be double-blind. However, there was a part which was not blinded - there were telephone interviews which recorded service use measures for economic analysis. These weekly telephone calls lasting about $10 \mathrm{~min}$ each had actually 3 purposes: to maintain engagement with the study, to screen for risks and to collect service use data for costing purposes. Patients were using psychotropic medication prescribed by their physicians. The main outcome measure was the score on the Work and Social Adjustment Scale (WSAS), and depression, anxiety, psychological functioning, costs and acceptability of the online process were also measured. The depressive symptomatology improved in both groups over the 6-week period, and most participants returned to work. There was no difference in any outcome measure between the MoodGYM and the control groups. However, the major problem of this study was the high attrition rate: data were collected for 637 subjects at baseline, 359 at 6 weeks (end of the intervention) and 251 subjects 12 weeks after baseline, that all in spite of the weekly telephone calls. The retention rate in this study was lower than in face-to-face studies. The results of this study raise the question whether online studies with no therapist involvement and no structured assignments are really effective. This was also impossible to judge due to another major limitation (in addition to the high attrition) - no real control group (e.g. TAU or waiting list). Apparently, we need to pay more attention to the design of online studies to be able to gauge their real usefulness. (This study was funded by the British Occupational Health Research Foundation.)

Smartphones have been used for monitoring patients to prevent relapse of mental illness for almost a decade [20]. In a recent study, Gustafson et al. [21] studied whether a smartphone application (A-CHESS - the Addiction Comprehensive Health Enhancement Support System) could provide effective continuing care and help recovery in alcohol use disorder patients. They enrolled 349 patients who met the DSM-IV criteria for alcohol dependence. The patients completed residential treatment in 5 different programs ( 3 US Midwest: CBT, motivational interviewing and psychoeducation, conducted almost entirely in group therapy; 2 US Northeast: group CBT and psychoeducation therapy, case management, supportive individual counseling based on motivational interviewing $+\mathrm{CBT}$ and 3 community Alcoholics Anonymous meetings per week). At the completion of the residential treatment, patients were randomized to the A-CHESS group or control group. Those in the A-CHESS group $(\mathrm{n}=170)$ received TAU + a smartphone with the ACHESS application for the 8-month intervention, and then TAU only during a 4-month follow-up. The control group $(\mathrm{n}=179)$ received TAU for 12 months. The ACHESS application had both static content (e.g. audioguided relaxation) and interactive features - e.g. if a patient neared a high-risk location such as a bar he/she used to frequent, the GPS initiated an alert asking if the patient wanted to be there. Patients were free to use the smartphones for personal purposes, only the use of A-CHESS was monitored. The main outcome measure was risky drinking days (number of days during which the patient's drinking in a 2 -hour period exceeded 4 standard drinks for men and 3 for women). Patients were asked to report their risky drinking in the previous 30 days on surveys taken 4,8 and 12 months after discharge from residential treatment. Patients in the A-CHESS group reported significantly fewer risky drinking days than those in the control group for the 8 months of the intervention and 4 months of follow-up ( 1.39 vs. 2.75 days; mean difference 1.37 ; 95\% CI $0.46-2.27 ; \mathrm{p}=0.003)$. Patients in the ACHESS group also reported a higher likelihood of consistent abstinence ( 51.9 vs. $39.6 \%, \mathrm{p}=0.03$ ). The important issue in the practicality of this effective intervention is the cost and reimbursement. The 8 months of A-CHESS cost about 597 USD (this included counselor time, administrator time, monthly fee for the data plan and the cost of the phone). (This study was funded by a grant from the US National Institute on Alcohol Abuse and Alcoholism.)

However - as I warned before [2] - in their review evaluating the current state of the science of research on the efficacy of computer-based therapies, Kiluk et al. [22] pointed out that the field has not yet reached the level of methodological quality equivalent to those required for behavioral therapy or pharmacotherapy studies. This seems obvious especially for the study of Phillips et al. [19]. (This review was supported in part by the National Institute on Drug Abuse and by VINS 1 Mental Illness Research, Education, and Clinical Center.)

\section{Issues in Treatment of Depression}

Boschloo et al. [23] explored whether baseline clinical factors, sociodemographics, childhood trauma, personality and life events predicted the 4 -year course (i.e. sustained recovery, temporary recovery and chronic course) of MDD. They also examined whether these associations were different for patients with chronic versus nonchron- 
ic MDD at baseline (i.e. simple staging of the disease). They examined how many of 514 patients with nonchronic depression progressed to chronic depression and the persistence of chronic MDD in the 253 patients with chronic depression. Patients received various psychological treatments, antidepressants and benzodiazepines. The data were derived from the Netherlands Study of Depression and Anxiety, an ongoing cohort study aimed at examining the long-term course and consequences of depressive and anxiety disorders in the population 18-65 years of age. The information about the course of illness was based on the Composite International Diagnostic Interview (done by phone), as well as the Life Chart method used at the 2- and 4-year follow-up assessment. At baseline, patients with chronic MDD had more severe depressive symptomatology, had more often a comorbid anxiety disorder, were more often treated with antidepressants, were older, had higher scores on childhood trauma and neuroticism, but lower scores on extraversion and consciousness, compared to the nonchronic MDD group. In the nonchronic MDD group at the baseline, 27.8\% developed a chronic disorder during the follow-up, while $53.0 \%$ of patients with chronic MDD at baseline had a persistent chronic disorder during the follow-up. Severity of MDD, childhood trauma and older age were important prognostic risk factors for a poor prognosis, independent of MDD chronicity at baseline. Importantly, an additional $31.5 \%$ of the nonchronic group at baseline suffered from residual symptoms at the follow-up - an important risk factor for relapse. Similarly, another $24.5 \%$ of the chronic MDD at baseline group suffered from residual symptoms at the follow-up. As the authors pointed out, the prospective course of MDD depended substantially on its chronicity at baseline. Another important finding was that recent negative life events were a significant risk factor for a poor prognosis of MDD in a subsample of patients with chronic MDD and high neuroticism at baseline. The study has several limitations, such as the lack of a strict definition of chronicity and a simple definition of staging of disease (chronic vs. nonchronic). Nevertheless, simple or complicated staging could be an important factor in treatment planning. Various risk factors, such as chronicity of illness, childhood trauma, recent life events and older age should be considered in staging and treatment selection. (This study was a part of the Netherlands Study of Depression and Anxiety conducted at several Dutch institutions, but the exact source of funding is not identified.)

As Psaros et al. [24] pointed out, the risks of untreated depression during pregnancy are significant and may in- clude poor maternal nutrition, inadequate weight gain, poor prenatal care, the use of tobacco, alcohol and other drugs of abuse and an increased risk for postpartum depression. Nevertheless, many women with remitted MDD discontinue antidepressant medication to avoid fetal drug exposure, thus increasing the risk of depression relapse. Thus, finding an alternative to antidepressant treatment during pregnancy is very important. Therefore, Psaros et al. [24] tested the effectiveness of CBT in preventing recurrence of depression in the highrisk population of women with a history of MDD who elected to discontinue antidepressant use during attempts to conceive. In their open preliminary study of CBT for the prevention of recurrence, they enrolled 12 women planning to get pregnant or early in pregnancy with remitted MDD on maintenance antidepressants with a plan to discontinue antidepressants for pregnancy. The women received 12 CBT sessions in the acute 16week phase and 3 booster CBT sessions in the 12-week booster phase. Participants were assessed monthly during the acute phase and then twice during the follow-up using the depression module of the Mini-International Neuropsychiatric Interview, Montgomery-Åsberg Depression Rating Scale, CGI Quality of Life Satisfaction Questionnaire and Hamilton Rating Scale for Depression (probably an overkill of evaluations), in addition to pregnancy status assessment. Over the 24 weeks of the trial, 9 women $(75 \%)$ did not restart antidepressants and did not relapse. Of the 3 women who restarted antidepressant treatment, 2 experienced a depressive relapse (within 5 and 10 weeks), and 1 woman restarted the antidepressant in the absence of mood episode within a week after completing her antidepressant taper. This small study suggests that CBT could be successfully used in women who stop antidepressant treatment while planning to get pregnant or starting their pregnancy. (The funding for this study is not stated, only personal funding from various pharmaceutical and other companies for some authors is noted.)

\section{Innovative Interventions}

As about $10 \%$ of children with antisocial behavior will develop antisocial personality disorder in adulthood, many have called for early intervention studies to find out whether early intervention can prevent the development of antisocial personality disorder. One such recommended early intervention of unknown efficacy is parent/parenting training. In order to examine the efficacy 
of parenting training, Scott et al. [25] conducted a followup after 4-10 years of two randomized controlled trials of group parenting training of parents of children with antisocial behavior. In the first trial, 94 families of children 3-7 years old with antisocial behavior were allocated to parent training and 26 families to usual management. Families allocated to parent training showed a large reduction in childhood antisocial behavior after treatment compared to controls, and the improvement was maintained at the 18-month follow-up. Parenting intervention included 9 sessions working with The Incredible Years basic video, which shows 'right' or 'wrong' ways of handling children. The usual management included supportive therapy for parents, and children received help in understanding why they may feel angry or frustrated and in exploring strategies to change this. Follow-up data were collected 5.6-10.5 years after the intervention (children were 9.4-17.2 years old). In the second trial, this time of high-risk 4- to 6-year-olds with elevated levels of antisocial behavior, 58 families received parental training using The Incredible Youth video and sessions on how to read with the child (altogether 15 sessions), while 51 control families were offered a telephone helpline manned by the same staff, advising them how to access regular services (only 7 families contacted this helpline). The follow-up for this study was 4.2-7.7 years (children were 9.2-13.1 years old). The primary outcome measures in both studies were the two elements of antisocial personality - antisocial behavior and antisocial character traits. In the first study, both elements of antisocial personality were improved in the early intervention group at the long-term follow-up compared with the control group. In addition, reading ability improved, and parental expressed emotions were warmer. Teacher-rated and self-rated antisocial behaviors were unchanged. Early intervention was not associated with improved long-term outcomes in the second study. These results suggest that some early interventions such as parental training may prevent the development of antisocial personality in adolescence and may improve academic performance in children, where such an intervention is indicated. This certainly needs parental involvement and willingness. The authors suggested the possibility of using a 'dental care model' - with children having frequent checkups for antisocial behavior and applying intervention when indicated. (This study was supported by a grant from the UK Healthcare Foundation.)

Supported employment plays an important role in rehabilitation and recovery of people with severe mental illness. It is effective in improving competitive work out- comes over 1-2 years (mostly in US studies). It has also been shown that supported employment is superior to other approaches, such as vocational rehabilitation. However, long-term effectiveness of supported employment has not been clearly established. Thus, Hoffmann et al. [26] conducted a randomized controlled trial comparing the long-term effectiveness of supported employment and vocational rehabilitation in Switzerland. Patients in the supported employment group $(n=46)$, once employed, received on-the-job training and follow-along support to facilitate job maintenance. In the event of job loss, support and assistance were provided in securing a new job. Patients in the vocational rehabilitation group $(\mathrm{n}=54)$ received prevocational training in sheltered workshops for 6-12 months, and then employable participants completed a 3- to 6-month training in a competitive job. All support stopped after that regardless of obtaining a competitive job. Competitive work and hospital admissions were tracked for 5 years, and interviews were conducted at 2 and 5 years to assess recovery and quality of life. Data of 39 patients in the supported employment group and 49 patients in the vocational rehabilitation group were analyzed at the 5-year follow-up. Participants in supported employment were more likely to obtain a competitive job than those in the vocational rehabilitation group (65 vs. $33 \%$ ), worked more hours and weeks, earned higher wages and had longer job tenures. They were also less likely to be hospitalized, had fewer psychiatric admissions and spent fewer days in the hospital. The social return on investment was higher in the supported employment group. Though the study was limited by small sample size and exclusion of some very impaired patients, the follow-up period was fairly long. Supported employment is an efficacious part of rehabilitation, useful in the long term and in various countries. (This study was supported by grants from the Swiss National Science Foundation, Federal Social Insurance Office, the Stanley Thomas Johnson Foundation, the Gottfried and Julia Bangerter-Rhyner Foundation, the Bank Vontobel Foundation, the Dosenbach-Waser Foundation and the Karl Mayer Foundation.)

\section{Disclosure Statement}

The author declares no conflict of interest. 


\section{References}

1 Balon R: Clinical factor 2010. Psychother Psychosom 2011;80:195-198.

-2 Balon R: Clinical factor 2011. Psychother Psychosom 2012;81:199-205.

3 Balon R: Clinical factor 2012. Psychother Psychosom 2013;82:204-212.

4 Balon R: Clinical factor 2013. Psychother Psychosom 2014;83:330-340.

5 Fava GA: The clinical factor. Psychother Psychosom 2011;80:1-3.

6 Manrique-Garcia E, Zammit S, Dalman C, Hemmingsson T, Andreasson S, Allenbeck P: Prognosis of schizophrenia on persons with and without history of cannabis use. Psychol Med 2014;44:2513-2521.

7 Harrow M, Jobe TH, Faull RN: Does treatment of schizophrenia with antipsychotic medications eliminate or reduce psychosis? A 20-year multi-follow-up study. Psychol Med 2014;44:3007-3016.

8 Jääskeläinen E, Juola P, Hirvonen N, MacGrath JJ, Saha S, Isohanni M, Veijola J, Miettunen J: A systematic review and meta-analysis of recovery in schizophrenia. Schizophr Bull 2013;39:1296-1306.

-9 Østergaard Christensen T, Vesterager L, Krarup G, Olsen BB, Melau M, Gluud C, Nordentoft M: Cognitive remediation combined with an early intervention service in first episode psychosis. Acta Psychiatr Scand 2014;130: 300-310.

10 Bowen S, Witkiewitz K, Clifasefi SL, Grow J, Chawla N, Hsu SL, Carroll HA, Harrop E, Collins SE, Lustyk K, Larimer ME: Relative efficacy of mindfulness-based relapse prevention, standard relapse prevention, and treatment as usual for substance use disorders. A randomized clinical trial. JAMA Psychiatry 2014;71:547-556.

11 Wiersma JE, Van Shaik DJF, Hoogendorn AW, Dekker JJ, Van HL, Schoevers RA, Blom MBJ, Maas K, Smit JH, McCullough JP Jr, Beekman ATF, Van Oppen P: The effectiveness of cognitive behavioral analysis system of psychotherapy for chronic depression: a randomized controlled trial. Psychother Psychosom 2014;83:263-269.
12 Hollon SD, DeRubeis RJ, Fawcett J, Amsterdam JD, Shelton RC, Zajecka J, Yount PR, Gallop R: Effect of cognitive therapy with antidepressant medications vs antidepressants alone on the rate of recovery in major depressive disorder. A randomized clinical trial. JAMA Psychiatry 2014;71:1157-1164.

13 Leichsenring F, Salzer S, Beutel ME, Herpertz S, Hiller W, Hoyer J, Huesing J, Joraschky P, Nolting B, Poehlmann K, Ritter V, Stangier U, Strauss B, Stuhldreher N, Tefikow S, Teismann T, Willutzki U, Wiltink J, Leibing E: Psychodynamic therapy and cognitive-behavioral therapy in social anxiety disorder: a multi-center randomized controlled trial. Am J Psychiatry 2013;170:759-767.

14 Leichsenring F, Salzer S, Beutel ME, Herpertz S, Hiller W, Hoyer J, Huesing J, Joraschky P, Nolting B, Poehlmann K, Ritter V, Stangier U, Strauss B, Tefikow S, Teismann T, Willutzki U, Wiltink J, Leibing E: Long-term outcome of psychodynamic therapy and cognitive-behavioral therapy in social anxiety disorder. Am J Psychiatry 2014;171:1074-1082.

-15 Herbst N, Voderholzer U, Thiel N, Schaub R, Knaevelsrud C, Stracke S, Hertenstein E, Nissen C, Kulz AK: No talking, just writing! Efficacy of an Internet-based cognitive behavioral therapy with exposure and response prevention in obsessive compulsive disorder. Psychother Psychosom 2014;83:165-175.

16 Andersson E, Steneby S, Karlsson K, Ljotsson B, Hedman E, Enander J, Kaldo V, Andersson G, Lindefors N, Rück C: Long-term efficacy of Internet-based cognitive behavior therapy for obsessive-compulsive disorder with or without booster: a randomized controlled trial. Psychol Med 2014;44:2877-2887.

17 Andersson E, Enander J, Andren P, Hedman E, Ljotsson B, Hursti T, Bergström J, Kaldo V, Lindefors N, Anderson G, Ruck C: Internetbased cognitive-behaviour therapy for obsessive-compulsive disorder: a randomized controlled trial. Psychol Med 2012;42:2193-2203.

18 Wagner B, Horn AB, Maercker A: Internetbased versus face-to-face cognitive-behavioral intervention for depression: a randomized controlled non-inferiority trial. J Affect Disord 2014;152-154:113-121.
19 Phillips R, Schneider J, Molosankwe I, Leese M, Foroushani PS, Grime P, McCrone P, Morriss R, Thornicroft G: Randomized controlled trial of computerized cognitive behavioural therapy for depressive symptoms: effectiveness and costs of a workplace intervention. Psychol Med 2014;44:741-752.

20 Spaniel F, Vohlidka P, Hrdlicka J, Kozeny J, Novak T, Motlova L, Cermak J, Bednarik J, Novak D, Höschl C: ITAREPS: information technology aided relapse prevention programme in schizophrenia. Schizophr Res 2008;98:312-317.

21 Gustafson DH, McTavish FM, Chich M-Y, Atwood AK, Johnson RA, Boyle MG, Levy MS, Driscoll H, Chisholm SM, Dillenburg L, Isham A, Shah D: A smartphone application to support recovery from alcoholism. A randomized clinical trial. JAMA Psychiatry 2014; $71 ; 566-572$.

22 Kiluk BD, Sugarman DE, Nich C, Gibbons CJ, Martino S, Rousanville BJ, Carroll KM: A methodological analysis of randomized clinical trials of computer-assisted therapies for psychiatric disorders: toward improved standards for an emerging field. Am J Psychiatry 2011;168:790-799.

23 Boschloo L, Schoevers RA, Beekman ATF, Smit JH, van Hemert AM, Penninx AWJH: The four-year course of major depressive disorder: the role of staging and risk factor determination. Psychother Psychosom 2014;83: 279-288.

24 Psaros C, Freeman M, Safren S, Barsky M, Cohen LS: Discontinuation of antidepressants during attempts to conceive. A pilot trial of cognitive behavioral therapy for the prevention of recurrent depression J Clin Psychopharmacol 2014;34:455-460.

25 Scott S, Briskman J, O’Connor TG: Early prevention of antisocial personality: long term follow-up of two randomized controlled trials comparing indicated and selective approaches. Am J Psychiatry 2014;171:649-657.

26 Hoffmann H, Jäckel D, Glauser S, Mueser KT, Kupper Z: Long-term effectiveness of supported employment: 5-year follow-up of a randomized controlled trial. Am J Psychiatry 2014;171;1183-1190. 